\title{
Ocean-tide parameters from the simultaneous long-period analysis of the orbits of Starlette and Stella
}

\author{
N. M. Harwood, G. G. Swinerd \\ Department of Aeronautics and Astronautics, University of Southampton, Southampton, Hants, SO17 1BJ, UK \\ Received: 28 June 1996 / Revised: 24 July 1996 / Accepted: 6 August 1996
}

\begin{abstract}
The orbits of two geodetic satellites, Starlette and Stella, have been analysed in order to determine ocean-tide parameters. The orbit of Starlette has been determined over a three-year period and Stella over a one-year period. Long-period analysis techniques have been used to determine the evolutions of the orbital inclination, eccentricity and right ascension of the ascending node for each satellite due to ocean tides. The ocean-tide parameters have been determined in a simultaneous fitting of the theoretical orbital variations to the observed variations. The results are compared with ocean-tide models.
\end{abstract}

\section{Introduction}

The geodetic satellites Starlette and Stella were launched by CNES on 6 February 1975 and 1 September 1993, respectively. The satellites are identical in form and each consist of a dense $U_{238}$ core in order to minimize nongravitational forces. They are fitted with corner-cube reflectors for the purpose of laser-ranging. The orbital inclination of Starlette in $49.8^{\circ}$, with a semi-major axis of $7335 \mathrm{~km}$ and an eccentricity of 0.02 (King-Hele et al., 1990). The corresponding parameters of Stella are $i=98.7^{\circ}, a=7177 \mathrm{~km}$ and $e=0.001$. The orbit of Starlette has been analysed extensively by various authors using short-arc orbital analysis methods in order to determine ocean-tide parameters (see for example Williamson and Marsh, 1985; Moore, 1987). Such short-arc analyses can, however, be problematic when long-period information is to be determined, since the orbit is effectively 'reset' at the beginning of each new orbital arc. In particular, it is difficult to obtain accurate values for long-period ocean-tide parameters and secular variations in the Earth's gravity field using such methods. To overcome this problem, Cheng et al. (1990) used a 3-year long-arc to determine ocean-tide parameters from the analysis of Starlette. The results showed some significant discrepancies in the recovered parameter values when compared with the results from short-arc analyses. Another long-term analysis by Harwood and Swinerd (1996) over a 2-year period utilized a novel long-period analysis technique (Harwood and Swinerd (1995) in order to determine the ocean tidal coefficients.

In this work the previous analysis of Harwood and Swinerd is much improved, principally by the addition of a second geodetic satellite, Stella, and the extension of the Starlette data set by 1 year. The orbits were determined using the SATAN package and subsequently analysed using the long-period analysis technique to find the orbital variations due to ocean tides. The orbital variations were then fitted simultaneously in order to determine the ocean-tide parameters. The principal aim is to obtain further information regarding the longperiod ocean tides together with some of the tidal sideband terms. The results from the analysis provide tests for global ocean-tide models and help improve our knowledge of the Earth's gravity-field variations.

\section{The orbits}

The orbits were determined using a modified version of the SATAN package, which was written by Sinclair and Appleby (1986) at the Royal Greenwich Observatory. The package was written to conform to Project MERIT standards (Melbourne et al., 1983). In the previous analysis by Harwood and Swinerd (1996) the orbit of Starlette was determined over a 2-year period between January 1991 and December 1992. In this analysis the orbits have been redetermined over a longer period (January 1991 to December 1993) using a different orbital arc length and a different combination of orbital parameters. The Stella orbits were determined over a 1-year period between January 1994 and December 1994. Where possible, consecutive 3-day arcs were used throughout in order to allow determination of the longterm evolution of the orbits. The arc-length is 
necessarily short so as to avoid aliasing of highfrequency tidal effects into the observed orbital variation. However, where the data became too sparse the orbital arc length was doubled to compensate. Since this happened infrequently, this did not present any significant problems in the subsequent analysis. One drag coefficient was computed per orbital arc to allow for drag modelling errors, together with an empirical along-track once-per-revolution acceleration. One solarradiation-pressure parameter was also determined per arc for each satellite, but these values were constrained to their expected values of $C_{R}=1.134$ for Starlette and 1.1 for Stella. A 1-month gap occurred in the Starlette data set during April 1992.

\section{Perturbations due to ocean tides}

\subsection{Ocean tides}

The global representation of the ocean tide may be written in terms of spherical harmonics as (Lambeck, 1977),

$\xi_{\beta}=\sum_{s=0}^{\infty} \sum_{t=0}^{s} \sum_{+}^{-} D_{\beta, s t}^{ \pm} \cos \left(2 \pi f_{\beta} T \pm t \lambda-\varepsilon_{\beta, s t}^{ \pm}\right) P_{s t}(\sin \phi)$,

where $\phi, \lambda$ are latitude and longitude, respectively, $P_{s t}(\sin \phi)$ is the Legendre function of degree $s$ and order $t$, and $T$ is mean solar time. The $D_{\beta, s t}^{ \pm}$and $\varepsilon_{\beta, s t}^{ \pm}$are the amplitude and phase, respectively, for each ocean tidal constituent, $\beta$. The frequency, $f_{\beta}$, may be written in terms of the six fundamental arguments of the Sun and Moon $\left(\varphi_{1}-\varphi_{6}\right)$ as used by Doodson (1921), and is given by,

$2 \pi f_{\beta} T=\sum_{i=1}^{6} n_{i} \varphi_{i}$.

This is conventionally expressed as a Doodson number, $n_{1} n_{2} n_{3} \cdot n_{4} n_{5} n_{6}$, where $n_{1}$ is always non-negative and the remainder are added to five. The gravitational potential due to the ocean tide constituent $\beta$ at a radial distance $r$ from the Earth's centre and outside the Earth is then given by,

$$
\begin{aligned}
U_{\beta}= & 4 \pi G R \rho_{w} \sum_{s=0}^{\infty} \sum_{t=0}^{s} \sum_{+}^{-} \frac{1+k_{s}^{\prime}}{2 s+1}\left(\frac{R}{r}\right)^{s+1} D_{\beta, s t}^{ \pm} \\
& \cos \left(2 \pi f_{\beta} T \pm t \lambda-\varepsilon_{\beta, s t}^{ \pm}\right) P_{s} \mathrm{t}(\sin \phi),
\end{aligned}
$$

where $G$ is the Universal constant of gravitation, $R$ is the mean equatorial radius of the Earth, $\rho_{\omega}$ is the density of seawater and $k_{s}^{\prime}$ is a load-deformation Love-number.

Several different definitions exist for the phase, and that defined above is related to that of Schwiderski (1983), $\gamma_{\beta, s t}^{+}$, by

$\varepsilon_{\beta, s t}^{+}=-\left(\gamma_{\beta, s t}^{+}-\frac{\pi}{2}+\chi_{\beta}\right)$,

where

$$
\chi_{\beta}= \begin{cases}0 & \text { zonal and semi-diurnal tides }(t=0,2) \\ \frac{\pi}{2} & \text { diurnal tides }(t=1) \text { and } H_{\beta}>0, \text { e.g. } K_{1} \\ -\frac{\pi}{2} & \text { diurnal tides }(t=1) \text { and } H_{\beta}<0,\end{cases}
$$

and $H_{\beta}$ is the Doodson amplitude for the constituent $\beta$ from Cartwright and Edden (1973). In this paper the phases are given in the Schwiderski form in order to maintain consistency with the ocean-tide models from the global gravity-field models.

\subsection{Perturbations in inclination, eccentricity and right ascension of the ascending node}

The rate of change of orbital inclination, $i$, due to each $D_{\beta, s t}^{ \pm}$and $\varepsilon_{\beta, s t}^{ \pm}$is given by (Lambeck, 1977),

$$
\begin{aligned}
\frac{d i}{d t}= & \frac{1}{\sqrt{1-e^{2}} n a^{2}}[(s-2 u) \cot i-t \csc i] \operatorname{Re} \\
& \left(j^{s-t+1} A_{\beta, s t u v}^{ \pm} \exp j \Phi_{\beta, \text { stuv }}^{ \pm}\right),
\end{aligned}
$$

where

$$
A_{\beta, s t u v}^{ \pm}=4 \pi G R \rho_{\omega} \frac{1+k_{s}^{\prime}}{2 s+1}\left(\frac{R}{a}\right)^{s+1} D_{\beta, s t}^{ \pm} F_{s t u}(i) G_{s u v}(e)
$$

and

$$
\begin{aligned}
\Phi_{\beta, s t u v}^{ \pm}= & 2 \pi f_{\beta} T-\varepsilon_{\beta, s t}^{ \pm} \pm[(s-2 u) \omega+(s-2 u+v) M \\
& +t(\Omega-\theta)] .
\end{aligned}
$$

Here, the orbital elements have their usual meaning, $j=\sqrt{-1}$. Re denotes the real part, $F_{s t u}$ is the inclination function of Allan (1973), $G_{\text {suv }}$ is the eccentricity function (Kaula, 1966) and $\theta$ the sidereal angle. The integers $u$ and $v$ are such that $u=0,1,2, \ldots s, v=-\infty, \ldots \infty$, and dominant terms occur when $s=2 u$ and $v=0$. Longperiod perturbations only occur when $s-2 u+v=0$. Also, since $\varphi_{1}$ contains the sidereal angle the orbital perturbations with periods greater than one day will only occur for the prograde tides $\left(D_{\beta, s t}^{+}, \varepsilon_{\beta, s t}^{+}\right)$and when $n_{1}=t$.

The rates of change of right ascension of the ascending node, $\Omega$, and eccentricity $e$ are given, for each pertinent $\beta, s, t, u, v$, by,

$$
\frac{d \Omega}{d t}=\frac{1}{n a^{2} \sqrt{1-e^{2}} \sin i} \operatorname{Re}\left(j^{s-t} \frac{\partial A_{\beta, s t u v}^{ \pm}}{\partial i} \exp j \Phi_{\beta, s t u v}^{ \pm}\right)
$$

and

$\frac{d e}{d t}=-\frac{\sqrt{1-e^{2}}}{n a^{2} e}(s-2 u) \operatorname{Re}\left(j^{s-t+1} A_{\beta, s t w v}^{ \pm} \exp j \Phi_{\beta, s t w v}^{ \pm}\right)$.

\subsection{Interaction of inclination and eccentricity perturbations with the secular nodal perturbation}

The long-period perturbations in the orbital inclination due to ocean tides induce changes in the secular nodal rate due to the $J_{2}$ zonal harmonic. A similar effect is caused by the change in the eccentricity. These indirect 
perturbations in the right ascension of the ascending node must be taken into account and are given approximately by (Kaula, 1966),

$\Delta \Omega=-\frac{3 n J_{2}}{2\left(1-e^{2}\right)^{2}}\left(\frac{R}{a}\right)^{2}\left(\frac{4 e \cos i}{1-e^{2}} \int \Delta e d t-\sin i \int \Delta i d t\right)$.

The integrals are taken over the total long-period changes in $i$ and $e$ due to ocean tides.

\section{Methodology}

The objective of the analysis is to evaluate the ocean-tide parameters $D_{\beta, s t}^{ \pm}, \varepsilon_{\beta, s t}^{ \pm}$by obtaining the long-term changes in the satellite orbit due to ocean tides. The parameters are determined by a least-squares fitting of the theoretical orbital variations given by Eqs. 5-7 to the observed variations. The orbits were determined using the SATAN code and a novel long-period analysis technique was used to determine the variations in the orbital elements due to ocean tides. In this method a comparison is made between the state vector (start position and velocity) of one orbital arc with the corresponding vector from the end of the previous orbit (the orbital arcs being consecutive). The difference between these two vectors contains information regarding the mismodelling of the long-period perturbations within the SATAN code. The full method has been described previously and will not be repeated here (Harwood and Swinerd, 1995).

\section{Perturbation modelling errors}

\subsection{Gravity field}

In order to determine the ocean-tide parameters it is necessary to have a complete and accurate perturbation model. The SATAN code contains such a model; however, mismodelling of some perturbations is inevitable, and this may result in aliasing of the resulting tidal coefficients. In the previous analysis it was found that gravity-field modelling errors are important. To account for this, corrections were found for the lowdegree zonal harmonics in the solutions for the oceantide parameters. In order to determine the long-period orbital variations due to the gravity-field modelling errors, Equations 3.76 of Kaula (1966) were used. A similar procedure is used here, and account is also taken of the time dependence of the zonal harmonics $\left(\dot{J}_{2}, \dot{J}_{3}\right)$. The short-period perturbations due to gravity field errors are largely accounted for by the determination of once per rev empirical decelerations. It was not possible to distinguish between the long-term effects due to errors in the $J_{2,2}$ tesseral harmonic and those due to the $S_{2}$ solar ocean tide since the resulting long-period orbital perturbations are indistinguishable. In this analysis a preliminary version of the JGM3 gravity field was used to determine the orbits, and so the results for $S_{2}$ are with respect to the JGM3 value of $J_{2,2}$.

\subsection{Air drag}

Air drag is another major source of error in the orbit determination and analysis process. This was largely accounted for in the orbit determination process by determining a drag coefficient per arc (usually every 3 days). Aliasing with tidal coefficients is anticipated to be small since the principal drag effect is in the along-track direction and will mainly affect the mean anomaly and semi-major axis. The along-track motion is, therefore, effectively being used to correct for the drag modelling errors. Severe drag mismodelling may, however, affect the long-period variation in the eccentricity with some consequent aliasing in the odd-degree long-period tidal coefficients. The drag model used for this analysis is CIRA72 (1972).

\subsection{Solid-tide model and atmospheric tides}

The effect of ocean tides are indistinguishable from those of solid Earth tides and are therefore relative to the particular solid-tide model used. The orbital model in the SATAN code conforms to MERIT standards and the solid-tide model is based on an abbreviated form of the Wahr model (Wahr, 1981). The $S_{2}$ atmospheric tide is not accounted for and so the results for this tide include the atmospheric effect.

\section{Treatment of tidal sidebands}

Each principal ocean tide consists of a central frequency and a number of smaller side-band terms. The sidebands are separated from the main terms by low frequencies which are typically different from the central term by a multiple of $\varphi_{5}$, the negative of the lunar perigee. One reason for performing long-period analyses is to obtain some information regarding these sidebands. To obtain accurate parameter values without additional information would, however, require analysis over at least half a cycle of the lunar perigee. Since only 3 years of Starlette data and 1 of Stella are utilized in this analysis, additional information is required to account for the side-bands. The procedure adopted was to assume that the tidal admittance at the side-band frequencies is identical to that at the central frequency. The side bands were not, however, removed from the recovered coefficient values, but solved for and constrained to a fraction, $f_{j}$, of the amplitudes of the central term for any particular tide $j$. The $f_{j}$ of the relevant terms were determined by examination of Table 2 of Cartwright and Edden (1973). Only significant sidebands were considered and ill-determined side-bands were dropped from the fittings. 


\section{Analysis}

\subsection{Method}

Dominant long-period changes in the orbital inclination occur only for the even-degree tidal harmonics and when $t \neq 0$ (Harwood and Swinerd, 1996). For the right ascension of the ascending node, dominant terms arise when $s$ is even, but now we have that $t=0,1,2$, and so the nodal perturbation is sensitive to the long-period tides. For the eccentricity, dominant perturbations arise for the odd-degree harmonics and for $t=0,1,2$. The resulting perturbations to a single orbital element due to tidal coefficients of different degree generally all have the same frequency. A linear combination of coefficients will therefore be obtained from the analysis of a single orbital element. Account must also be taken of the indirect nodal perturbations due to changes in the secular $J_{2}$ nodal rate which are in turn caused by changes in the inclination and eccentricity (Eq. 8). For these reasons the analyses were performed simultaneously for the inclination, eccentricity and node and for both satellites. In this manner it is possible to obtain good separation of the tidal coefficients of different degree and, at the same time, to account for the indirect effects. Trial fittings were, however, performed for the individual orbital elements and satellites in order to identify which terms were likely to be important in the final fitting.

\subsection{Solutions}

After performing the trial fittings for the individual orbital elements, a simultaneous fitting was attempted for all orbital elements and for both satellites. All major diurnal, semi-diurnal and long-period tides at degrees 2 to 6 were included in this fitting. Corrections were also found for the low-degree zonal harmonics $\left(J_{2}\right.$ and $\left.J_{3}\right)$ and their rates of change. After removal of excessively ill-fitting parameters, a measure of fit, $\varepsilon$, was determined for each individual orbital element. The measures of fit were then used to weight the data sets of the individual orbital variations in the subsequent fittings. Further runs were then performed, dropping poorly fitting parameters and introducing new ones where appropiate.
The time covered by the data sets did not permit separation of the 18.6-year tides from the rates of change of the low-degree zonal harmonics. The procedure adopted for the second-degree coefficients $\left(\dot{J}_{2}\right.$ and 18.6-year tide, $\left.s=2\right)$ was to constrain the 18.6year tide to equilibrium and $\dot{J}_{2}$ strongly to that of JGM2 (Nerem et al., 1994). The 18.6-year tide at $s=3$ and the rate of change of $J_{3}$ were subsequently dropped from the fitting since these were not well determined. This is likely to be due to the drag modelling errors.

After determination of a satisfactory fitting, an attempt was made to account for some of the principal side-bands. Six side-bands were found to be significant at degree 2, $\left(M_{f}, Q_{1}, O_{1}, K_{1}, M_{2}\right.$ and $\left.K_{2}\right)$ together with one at degree $3\left(K_{1}\right)$ and at one at degree $5\left(K_{1}\right)$. Illfitting data points were then relaxed in order to keep all weighted residuals below $2 \varepsilon$. The results for this best fit are given in Tables 1, 2 and 3 for $t=0,1$ and 2, respectively. The fittings (curves) to the data (points) for the Starlette inclination, eccentricity and right ascension of the ascending node over the period May 1992 to December 1993 are given in Figs 1, 2 and 3, respectively. The corresponding fittings for Stella are given in Figs. 4 6. The fittings for Starlette over the period January 1991 to March 1992 are given in Harwood and Swinerd (1996). The results from this analysis, and over this period, do not differ significantly from those of the previous analysis, with the exception of a small slope to the eccentricity variation. This is thought to be due to the improvements in the orbit determination and perturbation modelling processes (Sects. 2 and 5), and should result in more reliable values for the long-period tides at odd degree. However, since the 18.6-year tide was not determined at $s=3$ or $s=5$, it is likely that drag modelling errors are still present. Future analyses will require further investigation into such errors in order to determine improved values for the odd-degree tidal coefficients.

\section{Comparison with ocean-tide models}

A comparison is given in Tables 1 to 3 of the values of ocean-tide coefficients from this analysis with those from the comprehensive gravity-field model GEM-T3

Table 1. Long-period ocean-tide parameters from the final fitting $(\mathrm{a}=$ this analysis, $\mathrm{b}=\mathrm{GEM}-\mathrm{T} 3, \mathrm{c}=\mathrm{Cheng})$

\begin{tabular}{|c|c|c|c|c|c|c|c|c|}
\hline Tide & $\begin{array}{l}\text { Doodson } \\
\text { Number }\end{array}$ & $s$ & $\begin{array}{l}\text { Amplitude } \\
D_{2,0}^{+}(\mathrm{cm}) \\
\mathrm{a}\end{array}$ & $\mathrm{b}$ & $\mathrm{c}$ & $\begin{array}{l}\text { Phase } \\
\gamma_{2,0}^{+} \text {(Deg.) } \\
\mathrm{a}\end{array}$ & $\mathrm{b}$ & c \\
\hline & 055.565 & 2 & $1.56 \pm 0.07$ & & & $269.9 \pm 0.9$ & & \\
\hline \multirow[t]{2}{*}{$S_{a}$} & 056.554 & 2 & $2.05 \pm 0.03$ & $2.65 \pm 0.29$ & 2.83 & $30.3 \pm 0.6$ & $26.3 \pm 6.8$ & 39.0 \\
\hline & 056.554 & 3 & $2.33 \pm 0.29$ & $5.17 \pm 0.52$ & $1.98 \pm 0.36$ & $300.9 \pm 5.0$ & $314.0 \pm 5.8$ & $245.2 \pm 11.2$ \\
\hline \multirow[t]{4}{*}{$S_{s a}$} & 057.555 & 2 & $2.07 \pm 0.05$ & $1.61 \pm 0.30$ & $1.59 \pm 0.68$ & $282.3 \pm 1.0$ & $255.9 \pm 10.8$ & $253.3 \pm 5.2$ \\
\hline & 057.555 & 3 & $2.51 \pm 0.17$ & $0.53 \pm 0.51$ & $0.78 \pm 0.62$ & $6.8 \pm 2.6$ & $54.1 \pm 55.3$ & $69.2 \pm 6.5$ \\
\hline & 058.554 & 2 & $0.98 \pm 0.08$ & & & $164.6 \pm 3.1$ & & \\
\hline & 058.554 & 3 & $0.75 \pm 0.09$ & & & $297.0 \pm 5.7$ & & \\
\hline$M_{m}$ & 065.455 & 2 & $0.78 \pm 0.32$ & $0.84 \pm 0.27$ & $1.42 \pm 0.67$ & $179.7 \pm 17.0$ & $260.1 \pm 18.6$ & $245.5 \pm 7.5$ \\
\hline \multirow[t]{2}{*}{$M_{f}$} & 075.555 & 2 & $2.43 \pm 0.57$ & $2.05 \pm 0.28$ & $2.84 \pm 0.41$ & $280.1 \pm 9.5$ & $240.4 \pm 7.8$ & $242.1 \pm 25.3$ \\
\hline & 075.565 & 2 & $1.01 \pm 0.24$ & & & $280.1 \pm 9.5$ & & \\
\hline
\end{tabular}


Table 2. Diurnal ocean-tide parameters from the final fitting $(\mathrm{a}=$ this analysis, $\mathrm{b}=\mathrm{GEM}-\mathrm{T} 3, \mathrm{c}=\mathrm{Cheng})$

\begin{tabular}{|c|c|c|c|c|c|c|c|c|}
\hline Tide & $\begin{array}{l}\text { Doodson } \\
\text { Number }\end{array}$ & $s$ & $\begin{array}{l}\text { Amplitude } \\
D_{2,1}^{+}(\mathrm{cm}) \\
\mathrm{a}\end{array}$ & $\mathrm{b}$ & $\mathrm{c}$ & $\begin{array}{l}\text { Phase } \\
\gamma_{2,1}^{+} \text {(Deg.) } \\
\mathrm{a}\end{array}$ & $\mathrm{b}$ & $\mathrm{c}$ \\
\hline & 135.645 & 2 & $0.34 \pm 0.06$ & & & $0.8 \pm 8.1$ & & \\
\hline \multirow[t]{2}{*}{$Q_{1}$} & 135.655 & 2 & $1.78 \pm 0.29$ & & $0.72 \pm 0.22$ & $0.4 \pm 6.9$ & & $294.6 \pm 12.8$ \\
\hline & 145.545 & 2 & $0.33 \pm 0.05$ & & & $299.4 \pm 6.6$ & & \\
\hline \multirow[t]{2}{*}{$O_{1}$} & 145.555 & 2 & $1.78 \pm 0.23$ & $2.70 \pm 0.11$ & $2.66 \pm 0.23$ & $299.3 \pm 5.0$ & $314.5 \pm 2.4$ & $326.7 \pm 10.3$ \\
\hline & 145.555 & 4 & $0.88 \pm 0.24$ & $1.84 \pm 0.18$ & $2.25 \pm 0.31$ & $284.0 \pm 11.7$ & $282.5 \pm 5.4$ & $295.5 \pm 20.4$ \\
\hline \multirow[t]{4}{*}{$P_{1}$} & 163.555 & 2 & $1.20 \pm 0.05$ & $0.97 \pm 0.13$ & $0.99 \pm 0.07$ & $322.3 \pm 1.7$ & $313.9 \pm 7.5$ & $331.0 \pm 2.8$ \\
\hline & 163.555 & 3 & $1.67 \pm 0.12$ & $0.38 \pm 0.09$ & $0.86 \pm 0.03$ & $202.1 \pm 2.9$ & $6.5 \pm 14.5$ & $0.9 \pm 2.8$ \\
\hline & 163.555 & 4 & $0.68 \pm 0.05$ & $0.84 \pm 0.18$ & $0.78 \pm 0.03$ & $274.7 \pm 3.3$ & $256.1 \pm 12.1$ & $267.1 \pm 8.4$ \\
\hline & 163.555 & 5 & $1.78 \pm 0.10$ & $0.76 \pm 0.20$ & & $207.6 \pm 2.4$ & $122.9 \pm 14.3$ & \\
\hline \multirow{7}{*}{$K_{1}$} & 165.555 & 2 & $2.54 \pm 0.05$ & $2.78 \pm 0.12$ & $2.68 \pm 0.09$ & $328.1 \pm 0.8$ & $325.1 \pm 2.6$ & $324.9 \pm 6.9$ \\
\hline & 165.555 & 3 & $2.67 \pm 0.10$ & $0.79 \pm 0.09$ & $1.41 \pm 0.10$ & $331.7 \pm 1.5$ & $13.6 \pm 6.9$ & $346.5 \pm 7.1$ \\
\hline & 165.555 & 4 & $2.59 \pm 0.04$ & $2.39 \pm 0.17$ & $2.59 \pm 0.08$ & $254.6 \pm 0.6$ & $257.2 \pm 4.2$ & $253.8 \pm 12.8$ \\
\hline & 165.555 & 5 & $2.21 \pm 0.07$ & $2.23 \pm 0.20$ & & $93.3 \pm 1.4$ & $107.8 \pm 4.9$ & \\
\hline & 165.565 & 2 & $0.34 \pm 0.03$ & & & $325.7 \pm 3.9$ & & \\
\hline & 165.565 & 3 & $0.37 \pm 0.04$ & & & $331.7 \pm 4.1$ & & \\
\hline & 165.565 & 5 & $0.30 \pm 0.03$ & & & $97.1 \pm 4.8$ & & \\
\hline
\end{tabular}
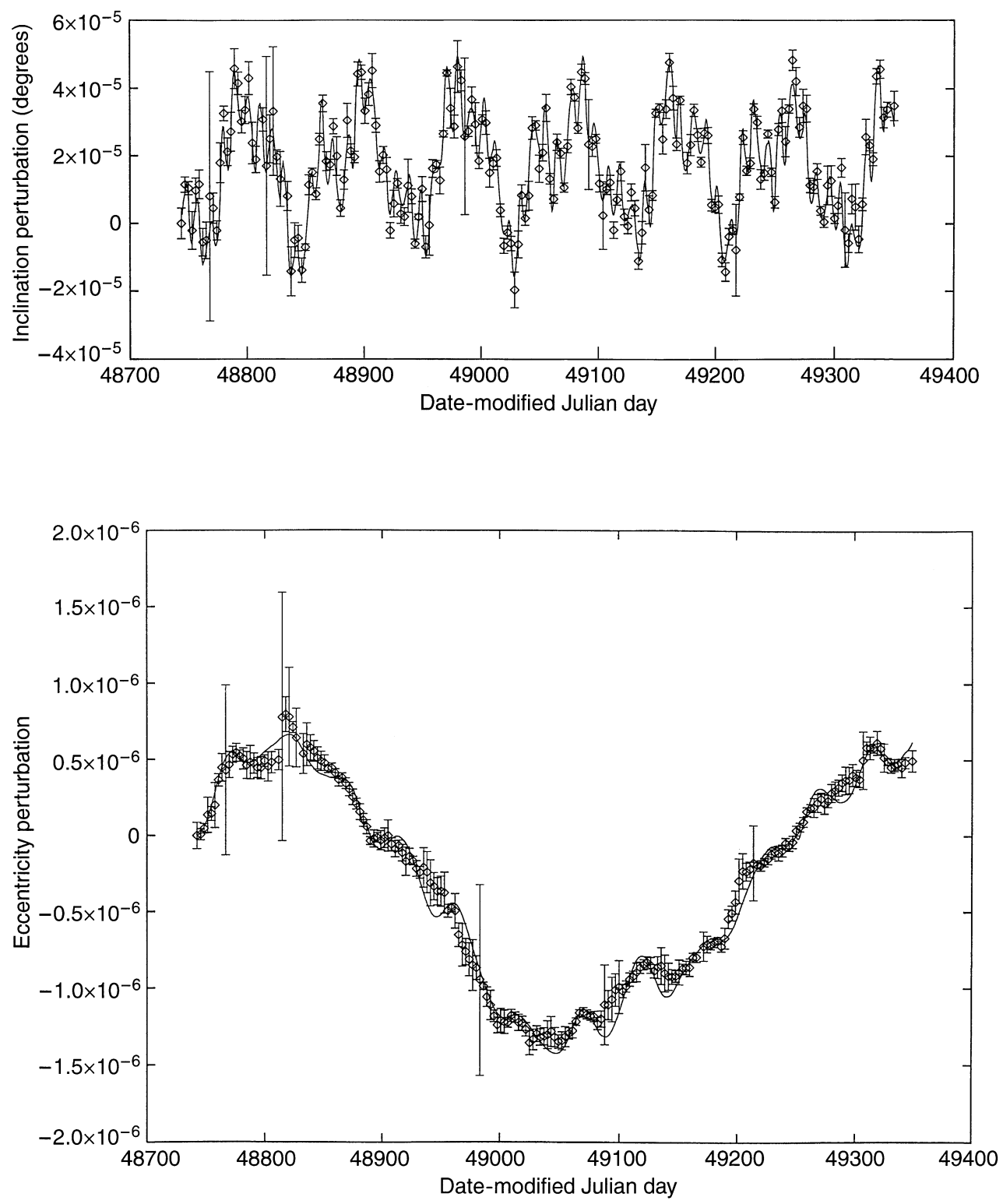

Fig. 1. The change in inclination of Starlette due to ocean tides from the simultaneous fitting (May 1992-December 1993)
Fig. 2. The change in eccentricity of Starlette due to ocean tides from the simultaneous fitting (May 1992December 1993) 


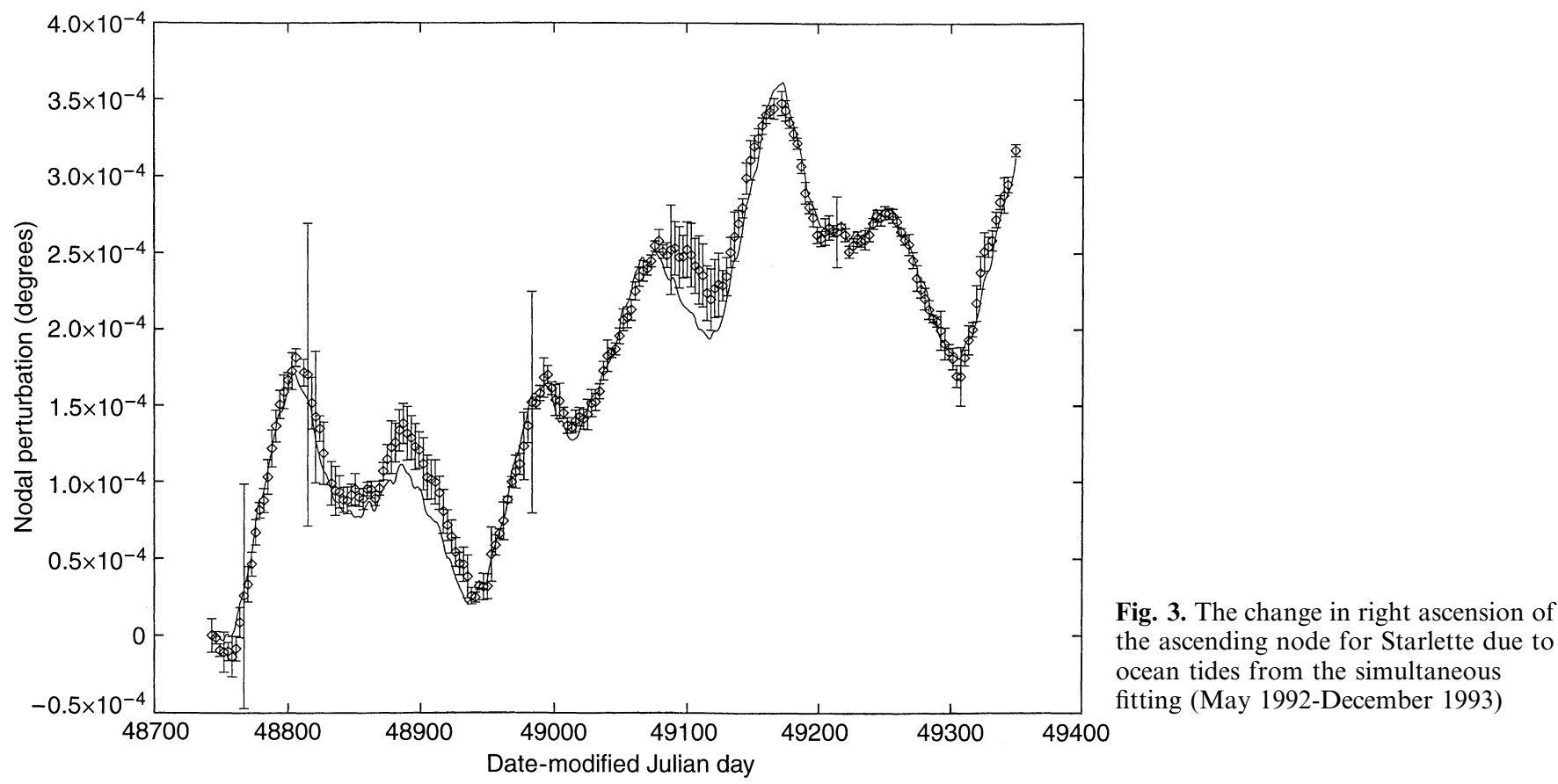

(Lerch et al., 1992) and from the analysis by Cheng (1992). For convenience the comparisons are divided into degrees 0,1 and 2 .

\subsection{Long-period tides $(t=0)$}

Particular consideration is given to the long-period tides, since the analysis technique used here is particularly sensitive to these tides. For the yearly tide $S_{a}$, the amplitude from this analysis at $s=2$ is smaller than those of GEM-T3 and Cheng (1990) although the phases generally agree. For $s=3$ the amplitude is in agreement with that of Cheng (to within the sum of the standard deviations), but not with GEM-T3. For the phases, however, the situation is reversed, the value from this analysis agrees with GEM-T3 but not with Cheng. For $S_{s a}$ the amplitude from this analysis at $s=2$ is somewhat larger than those of GEM-T3 and Cheng but agree to within the sum of the standard deviations. The phase is also slightly different. The values for $s=3$ do not, however, agree. It is noteworthy that the formal

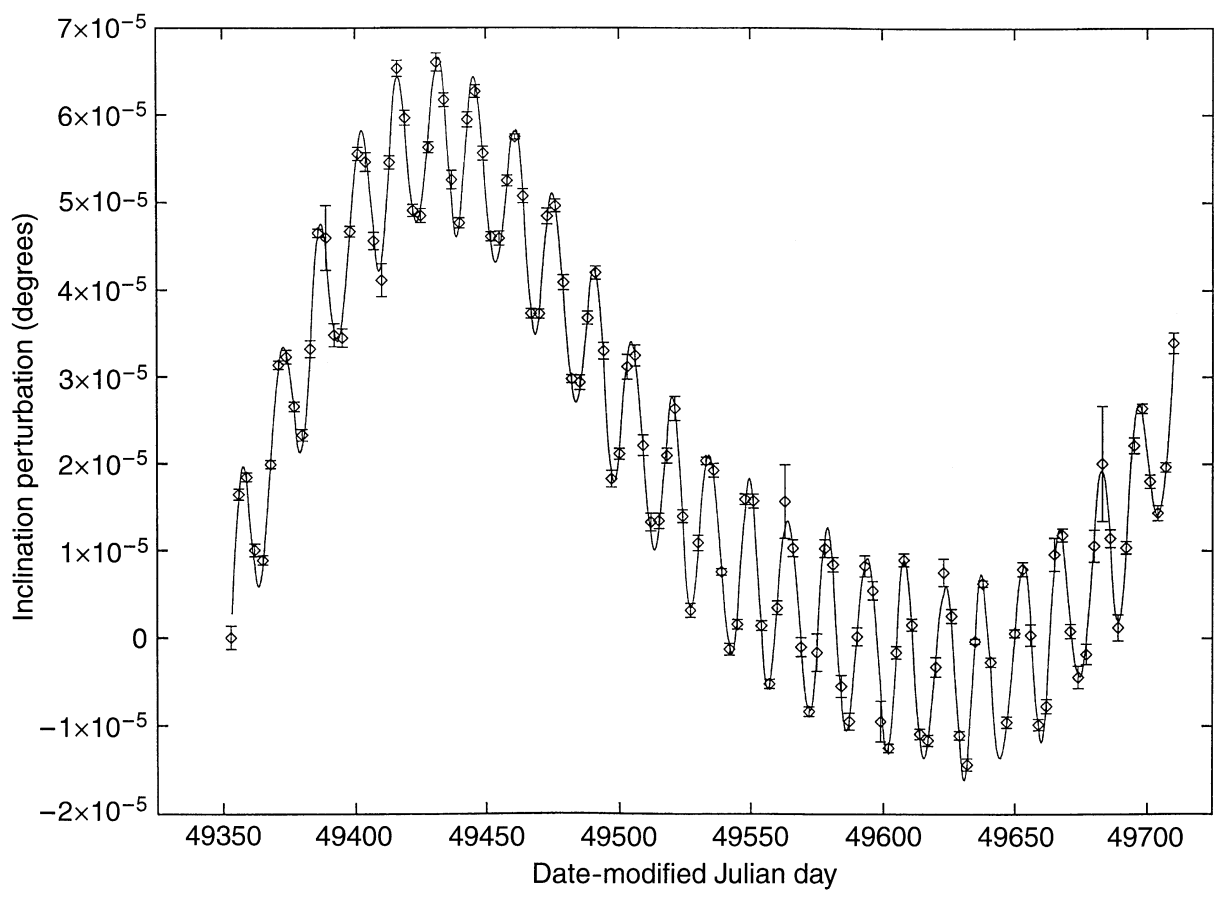

Fig. 4. The change in inclination of Stella due to ocean tides from the simultaneous fitting 


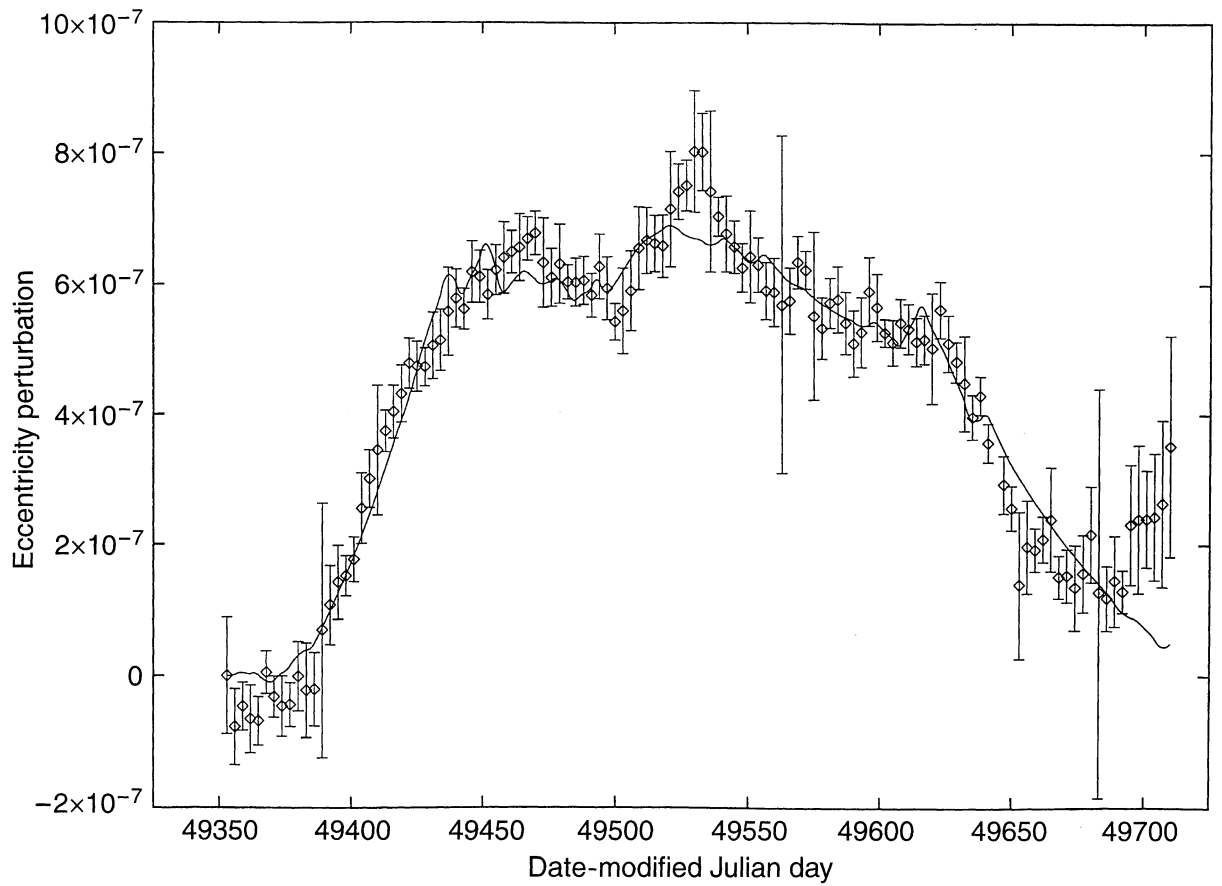

Fig 5. The change in eccentricity of Stella due to ocean tides from the simultaneous fitting accuracies of the coefficients from this analysis are in general better than those of GEM-T3 and Cheng (nominally ten times more accurate for $S_{s a}$ at degree 2).

The monthly and twice-monthly tides are particularly difficult to obtain accurate values for. The amplitudes for $M_{m}$ and $M_{f}$ are, however, in agreement with those of GEM-T3 and Cheng to within the quoted levels of uncertainty. For $M_{f}$ the value is approximately halfway between that of GEM-T3 and Cheng, and for $M_{m}$ our value is close to that of GEM-T3. There is, however, some disagreement between the phases from this analysis and those of GEM-T3 and Cheng, and this is particularly true for $M_{m}$. Results from satellite altimeter measurements (Ray and Cartwright, 1994) give $D_{2,0}^{+}=1.63 \pm 0.32 \mathrm{~cm}, \gamma_{2,0}^{+}=254.2^{\circ} \pm 12.0^{\circ}$ for $M_{f}$, and $\mathrm{D}_{2,0}^{+}=0.87 \pm 0.30 \mathrm{~cm}, \gamma_{2,0}^{+}=259.1^{\circ} \pm 21.0^{\circ}$ for $M_{m}$. The amplitudes from the orbital analyses for $M_{f}$ are all somewhat larger than the altimeter values and are generally in agreement to within the sum of the standard deviations (with the possible exception of Cheng's value). The phase from this analysis is slightly at odds with the altimeter results and those of GEM-T3 and Cheng. The $M_{m}$ altimeter amplitude is in good agreement with that from this analysis and GEM-T3. For the

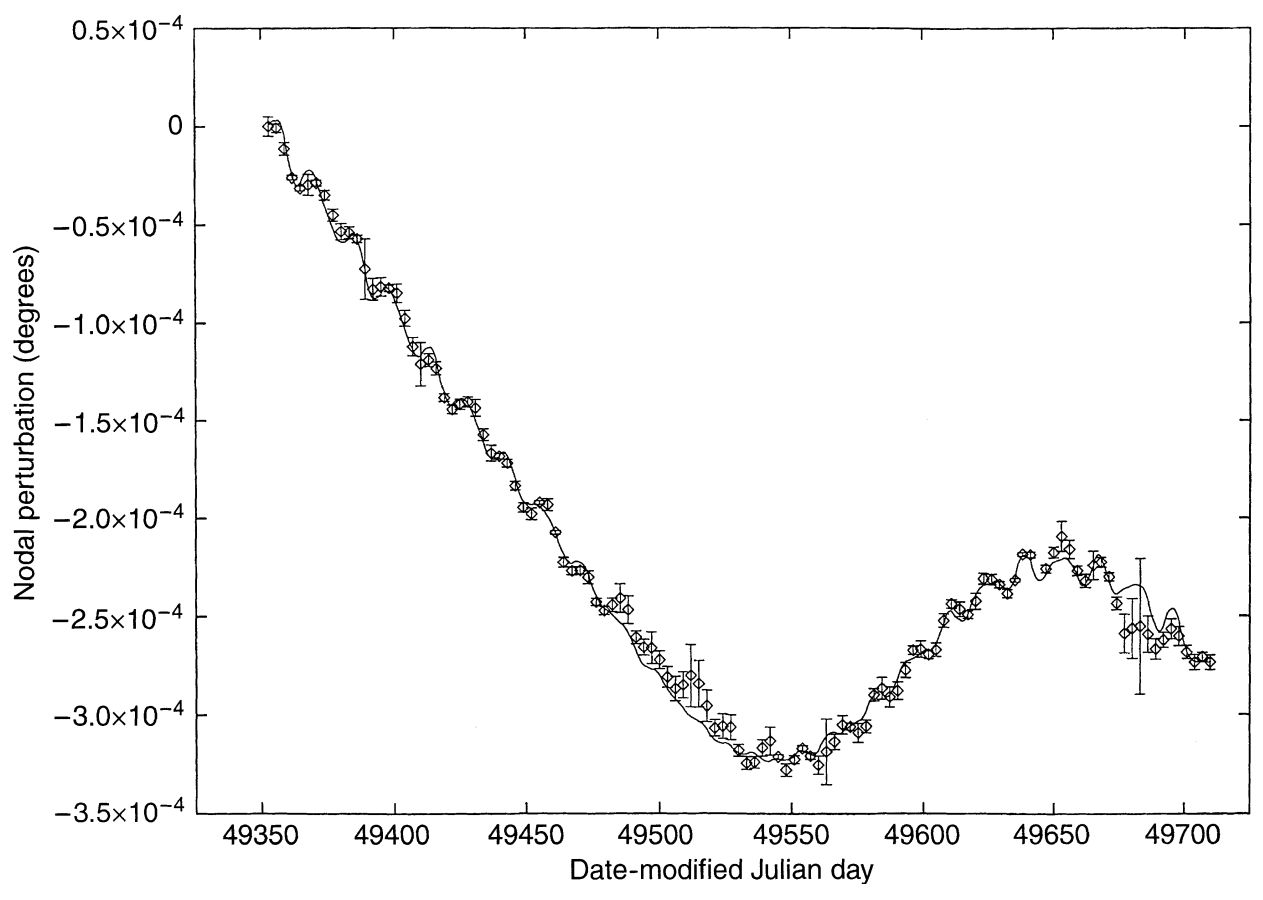

Fig 6. The change in right ascension of the ascending node of Stella due to ocean tides from the simultaneous fitting 
Table 3. Semi-diurnal ocean-tide parameters from the final fitting $(\mathrm{a}=$ thisanalysis, $\mathrm{b}=\mathrm{GEM}-\mathrm{T} 3, \mathrm{c}=\mathrm{Cheng})$

\begin{tabular}{|c|c|c|c|c|c|c|c|c|}
\hline Tide & $\begin{array}{l}\text { Doodson } \\
\text { Number }\end{array}$ & $s$ & $\begin{array}{l}\text { Amplitude } \\
D_{2,2}^{+}(\mathrm{cm}) \\
\mathrm{a}\end{array}$ & $\mathrm{b}$ & c & $\begin{array}{l}\text { Phase } \\
\gamma_{2,2}^{+} \text {(Deg.) } \\
\mathrm{a}\end{array}$ & $\mathrm{b}$ & $\mathrm{c}$ \\
\hline \multirow[t]{4}{*}{$N_{2}$} & 245.655 & 2 & $1.08 \pm 0.07$ & $0.69 \pm 0.06$ & $0.92 \pm 0.05$ & $342.2 \pm 2.6$ & $334.3 \pm 4.7$ & $329.3 \pm 5.5$ \\
\hline & 245.655 & 4 & $0.62 \pm 0.07$ & $0.23 \pm 0.04$ & $0.13 \pm 0.05$ & $171.0 \pm 4.5$ & $140.2 \pm 10.2$ & $148.2 \pm 2.9$ \\
\hline & 245.655 & 6 & $0.17 \pm 0.08$ & $0.07 \pm 0.05$ & & $18.8 \pm 17.8$ & $358.0 \pm 36.7$ & \\
\hline & 255.545 & 2 & $0.12 \pm 0.03$ & & & $316.0 \pm 11.5$ & & \\
\hline \multirow[t]{3}{*}{$M_{2}$} & 255.555 & 2 & $3.11 \pm 0.06$ & $3.31 \pm 0.04$ & $3.22 \pm 0.06$ & $318.0 \pm 0.8$ & $321.1 \pm 0.7$ & $319.3 \pm 2.4$ \\
\hline & 255.555 & 4 & $0.80 \pm 0.05$ & $0.98 \pm 0.04$ & $1.15 \pm 0.06$ & $113.7 \pm 2.7$ & $125.8 \pm 2.3$ & $120.6 \pm 4.3$ \\
\hline & 255.555 & 6 & $0.43 \pm 0.05$ & $0.39 \pm 0.04$ & & $325.7 \pm 5.0$ & $317.2 \pm 6.4$ & \\
\hline$L_{2}$ & 265.455 & 2 & $0.08 \pm 0.02$ & & & $312.5 \pm 9.9$ & & \\
\hline$T_{2}$ & 272.556 & 2 & $0.02 \pm 0.01$ & $0.05 \pm 0.04$ & $0.03 \pm 0.06$ & $203.1 \pm 15.7$ & $321.1 \pm 49.1$ & $13.1 \pm 2.4$ \\
\hline \multirow[t]{5}{*}{$S_{2}$} & 273.555 & 2 & $0.57 \pm 0.03$ & $0.78 \pm 0.02$ & $1.20 \pm 0.11$ & $291.2 \pm 2.2$ & $301.0 \pm 2.9$ & $315.7 \pm 11.3$ \\
\hline & 273.555 & 3 & $0.21 \pm 0.06$ & $0.29 \pm 0.03$ & $0.23 \pm 0.04$ & $190.7 \pm 12.1$ & $223.1 \pm 6.3$ & $191.6 \pm 1.8$ \\
\hline & 273.555 & 4 & $0.46 \pm 0.02$ & $0.36 \pm 0.04$ & $0.32 \pm 0.06$ & $101.5 \pm 1.6$ & $94.0 \pm 5.9$ & $89.1 \pm 6.2$ \\
\hline & 273.555 & 5 & $0.07 \pm 0.04$ & $0.16 \pm 0.03$ & & $46.1 \pm 22.4$ & $17.0 \pm 11.3$ & \\
\hline & 273.555 & 6 & $0.12 \pm 0.02$ & $0.17 \pm 0.04$ & & $198.6 \pm 6.0$ & $276.8 \pm 13.3$ & \\
\hline \multirow[t]{4}{*}{$K_{2}$} & 275.555 & 2 & $0.49 \pm 0.04$ & $0.34 \pm 0.04$ & $0.29 \pm 0.06$ & $301.8 \pm 3.0$ & $315.9 \pm 6.6$ & $315.9 \pm 2.5$ \\
\hline & 275.555 & 4 & $0.07 \pm 0.01$ & $0.15 \pm 0.04$ & $0.10 \pm 0.05$ & $143.5 \pm 8.4$ & $105.5 \pm 14.1$ & $97.0 \pm 4.1$ \\
\hline & 275.555 & 6 & $0.13 \pm 0.01$ & $0.04 \pm 0.04$ & & $346.7 \pm 3.2$ & $358.3 \pm 50.0$ & \\
\hline & 275.565 & 2 & $0.36 \pm 0.04$ & & & $337.6 \pm 4.0$ & & \\
\hline
\end{tabular}

phase, the agreement is better with those of GEM-T3 and Cheng's value than with this analysis (which differs from all three).

\subsection{Diurnal tides $(t=1)$}

For the diurnal tides $P_{1}$ and $K_{1}$ at even $s$ there is good agreement between the results of this analysis and those of GEM-T3 and Cheng. There is, however, a significant spread in the values of the odd-degree coefficients, with the exception of $D_{5,1}^{+}$for $K_{1}$, where the results of this analysis are in very good agreement with GEM-T3. For $O_{1}$ (even degree only) there are significant discrepancies in amplitude between these results and those of GEMT3 and Cheng, but the phases are in general agreement. The value for $Q_{1}(s=2)$ differs from that of Cheng, but account must be taken of the fact that only one degree of harmonic coefficient has been solved for in this analysis. Preliminary Topex/Poseidon altimetry results give $D_{2,1}^{+}=2.25 \mathrm{~cm}, \gamma_{2,1}^{+}=317.4^{\circ}$ for $O_{1}$, and $D_{2,1}^{+}=2.68 \mathrm{~cm}, \gamma_{2,1}^{+}=325.6^{\circ}$ for $K_{1} \quad$ (Schrama and Ray, 1994).

\subsection{Semi-diurnal tides $(t=2)$}

There is generally good agreement between the results from this analysis and those of GEM-T3 and Cheng for the semi-diurnal tides. Preliminary Topex/Poseidon results given $D_{2,2}^{+}=3.26 \mathrm{~cm}, \gamma_{2,2}^{+}=319.3^{\circ}$ for $M_{2}$ and $D_{2,2}^{+}=1.10 \mathrm{~cm}, \gamma_{2,2}^{+}=315.1^{\circ}$ for $S_{2}$ (account must be taken of the fact that the satellite orbital analysis results include the effects of the atmospheric tide on $S_{2}$ ). Cartwright and Ray (1991) give $D_{2,2}^{+}=3.45 \mathrm{~cm}$, $\gamma_{2,2}^{+}=318.4^{\circ}$ for $M_{2}$, and $D_{2,2}^{+}=1.16 \mathrm{~cm}, \gamma_{2,2}^{+}=323.8^{\circ}$ for $S_{2}$.

\section{Conclusions}

Ocean-tide parameters have been determined from the long-period analyses of the orbits of Starlette and Stella. The results show some significant discrepancies in the values for the long-period tides when compared with those of other models. The results from this analysis are, however, nominally more accurate than those of the models, and the method used here is particularly sensitive to the long-period tides. Agreement for the diurnal and semi-diurnal tides is good. The multisatellite analysis has allowed the determination of more tidal coefficients than in the analysis by Cheng (1990) and the previous analysis by Harwood and Swinerd (1996) (without having to constrain the results to any particular model). As such, the results from this multisatellite analysis, including Stella data, show a significant improvement in those determined using Starlette data alone. An extension of the data set to much longer time-period, together with the inclusion of more satellites, should allow a further substantial improvement over these results and for information to be obtained regarding the time dependence of the lowdegree zonal harmonics.

Acknowledgements. The authors wish to thank Dr. Andrew Sinclair and Graham Appleby of the Royal Greenwich Observatory, who produced and provided the SATAN package.

\section{References}

Allan, R. R., Satellite resonance with longitude-dependent gravityIII. Inclination changes for close satellites, Planet. Space Sci, 21, 201-225, 1973.

Cartwright, D. E., and A. C. Edden, Corrected tables of tidal harmonics, Geophys. J. R. Astron. Soc. 33, 253-264, 1973.

Cartwright, D. E., and R. D. Ray, Energetics of global ocean tides from Geosat altimetry, J. Geophys. Res. 96, 16897-16912, 1991. 
Cheng, M. K., C. K. Shum, R. J. Eanes, B. E. Schutz, and B. D. Tapley, Long-period perturbations in Starlette orbit and tide solution, J. Geophys. Res., 95, 8723-8736, 1990.

CIRA72, COSPAR International Reference Atmosphere. Akadamie, Berlin, 1972.

Doodson, A. T., The harmonic development of the tide generating potential, Proc. R. Soc. Lond., A100, 305-329, 1921.

Harwood, N. M, and G. G. Swinerd, Long periodic and secular perturbations to the orbits of Explorer 19 and Lageos due to direct solar radiation pressure, Celestial Mech., 62, 81-92, 1995.

Harwood, N. M, and G. G. Swinerd, Determination of ocean tidal parameters from the long-period analysis of the orbit of Starlette, Planet. Space Sci., 44, 107-115, 1996.

Kaula, W. M., Theory of Satellite Geodesy, Blaisdell, Waltham, M. A, 1966.

King-Hele, D. G., D. M. C. Walker, A. N. Winterbottom, J. A. Pilkington, H. Hiller, and G. E. Perry, The R. A. E. Table of Earth Satellites 1957-1989, R. A. E., Farnborough, 1990.

Lambeck, K., Tidal dissipation in the oceans: astronomical, geophysical and oceanographic consequences, Phil. Trans. R. Soc. Lond., A287, 545-593, 1977.

Lerch, F. J., R. S. Nerem, B. H. Putney, T. L. Felsentreger, B. V. Sanchez, S. M. Klosko, G. B. Patel, R. G. Williamson, D. S. Chinn, J. C. Chon, K. E. Rachlin, N. L. Chandler, J. J. McCarthy, J. A. Marshall, S. B. Luthcke, D. W. Pavlis, J. W. Robbins, S. Kapoor and E. C. Pavlis, Geopotential models of the Earth from satellite tracking, altimeter and surface gravity observations: GEM-T3 and GEM-T3S, NASA Technical Memorandum 104555, 1992.
Melbourne, W., R. Anderle, M. Feissel, R. King, D, McCarthy, D. Smith, B. Tapley, and R. Vicente, Project MERIT standards, United States Naval Observatory Circular No. 167, Washington, D. C., U. S. Naval Observatory, 1983.

Moore, P., Ocean tidal parameters from Starlette data, Bull. Gèod., 61, 223-234, 1987.

Nerem, S. R., F. J. Lerch, J. A. Marshall, E. C. Pavlis, B. H. Putney, B. D. Tapley, R. J. Eanes, J. C. Rief, B. E. Schutz, C. K. Shum, M. M Watkins, S. M. Klosko, J. C. Chan, S. B. Luthcke, G. B. Patel, N. K. Pavlis, R. G. Williamson, R. H. Rapp, R. Biancale and F. Nouel, Gravity model development for Topex/ Poseidon: Joint Gravity Models 1 and 2. J. Geophy. Res., 99, 24421-24447, 1994.

Ray, R. D., and D. E. Cartwright, Satellite altimeter observations of the $M_{f}$ and $M_{m}$ ocean tides, with simultaneous orbit corrections, Geophys. Monogr. 82, Volume 17, 1994.

Schrama, E. J. O., and R. D. Ray, A preliminary tidal analysis of Topex/Poseidon altimetry, J. Geophys. Res., 99, 24799-24808, 1994.

Schwiderski, E. W., Atlas of ocean tidal charts and maps, part 1: The semi-diurnal principal lunar tide $M_{2}$, Mar. Geod. 6, 219 265, 1983.

Sinclair, A. T., and G. M. Appleby, S.L.R. Tech. Note No. 9, R.G.O. Cambridge, 1986.

Wahr, J. M., Body tides on an elliptical, rotating, elastic and ocean-less Earth, Geophys. J. Roy. Astron. Soc., 64, 677-703, 1981.

Williamson, R. G., and J. G. Marsh, Starlette Geodynamics: The Earth's tidal response, J. Geophys. Res., 90, 9346-9352, 1985. 